\title{
Comparison between refraining from using neuromuscular blockade and using neuromuscular blockade with sugammadex in perioperative management of the patients with myasthenia gravis
}

\author{
Fujita Y., Kajiura T., Akahori T., Hashimoto A., Sato Y., Fujiwara Y. \\ Summary: \\ ${ }^{1}$ Department of Anesthesiology, Aichi Medical University School of Medicine
}

We concluded that compared as management of using no MR, patients with MG using MR and sugammadex could obtain higher success rate of extubation in operating room. Therefore, we thought that sugammmadex could obtain enough recovery from muscle relaxation in patients with MG because of $100 \%$ success rate of extubation in operating room. However, there were $3 / 41(7.3 \%)$ patients who were intubated again and needed artificial respiratory management in sugammadex group

Background and Goal of Study:

Before use of sugammadex, maintaining anesthesia for myasthenia gravis (MG) with minimal muscle relaxants (MR) was common. Recently case series of successful management using MR and sugammadex in patients with MG have been reported. However, comparison of safety for perioperative management between refraining from using MR ( $\mathrm{N}$ group) and using MR with sugammadex ( $\mathrm{S}$ group) is not clear. We demonstrated that the success rate of extubation in operating room for thymectomy in patients with MG without treating MR was $71.7 \%$ (43/60) [95\% confident interval (CI): 65.9-77.5\%]. In addition, the number of cases of impossible extubation requiring ventilation on that day was three $(3 / 60)$ in the $\mathrm{N}$ group (2015, J Anesth.). We retrospectively compared the success rate of extubation in operating room and postoperative respiratory condition between $\mathrm{N}$ and $\mathrm{S}$ group.

Materials and Methods:

As for $\mathrm{N}$ group, before using sugammadex in Japan, all 60 patients diagnosed with MG who underwent surgery under general anaesthesia between January 2004 and March 2010 were eligible. As for $\mathrm{S}$ group, all 41 counterparts between April 2010 and November 2015 patients were compared. We investigated success rate of extubation in operating room and perioperative complications.

Results:

In group $\mathrm{N}$ the success rate was $71.7 \%(43 / 60)$ [95 \% CI: 65.9 $77.5 \%$ ]. In group S the success rate was $100 \%$ [95\%CI: $94-$ $100 \%]$. The success rate of extubation in operating room in group $\mathrm{S}$ is higher than that in group $\mathrm{N}(\mathrm{P}<0.001)$. However, there were $3 / 41(7.3 \%)$ patients who were intubated again and needed artificial respiratory management. This rate was not significant compared with the rate of impossible extubation requiring ventilation on that day $(3 / 60)$ in the $\mathrm{N}$ group $(\mathrm{P}=0.684)$

Discussion:

We had some concerns. First, the background was different between the groups. With this observational study, in non MR group MG symptom was more severe. Second, the anesthesia management was progressing. However, effect of anesthesia management was limited. Therefore, we thought that, even in MG patients, reverse effect of sugammadex was enough strong and we could use MR safely in this study and recent literatures. We could use MR with sugammadex in MG patient, carefully.
Table 1 Baseline Characteristics of the Patients

\begin{tabular}{lrrr} 
& non MR & sugammadex & P value \\
& \multicolumn{1}{c}{$\mathrm{n}=60$} & $\mathrm{n}=41$ & \\
\hline male/female (cases) & $26 / 34$ & $22 / 19$ & \\
age (year) & $46.7 \pm 13.1$ & $57.1 \pm 16.2$ & $<0.01$ \\
body weight (kg) & $58.3 \pm 14.2$ & $61.7 \pm 15.3$ & 0.251 \\
height (cm) & $161.6 \pm 8.5$ & $160.9 \pm 8.3$ & 0.535 \\
BMI (kg/m²) & $22.1 \pm 3.9$ & $23.8 \pm 4.2$ & 0.344 \\
\%VC & $102.6 \pm 17.7$ & $105.3 \pm 15.3$ & 0.5 \\
\%FEV1.0 & $84.2 \pm 10.5$ & $75.2 \pm 17.4$ & 0.0097 \\
\hline
\end{tabular}

Osserman classification

$\left.\begin{array}{rrr}\mathrm{I} & 22 & 37 \\ \mathrm{IIa} & 17 & 2 \\ \mathrm{IIb} & 19 & 2 \\ \mathrm{III} & 2 & 0\end{array}\right)<0.01$

Table 2 Anesthetic management and extubation

non MR sugammadex $p$ value

$\mathrm{n}=60$ $\mathrm{n}=41$

anesthesia time (min) operation time (min)

$225.0 \pm 121.0 \quad 296.8 \pm 132.5 \quad<0.01$
extubation in operation room $162.6 \pm 116.2203 .8 \pm 106.3$

$<0.01$ anesthesia (cases)

TIVA with propofol $49(74 \%)$ $41(100 \%)$ $<0.01$

\begin{tabular}{rrrr} 
anesthesia (cases) & & & \\
TIVA with propofol & $1(1.7 \%)$ & $29(70.7 \%)$ & \\
GOS & $37(61.7 \%)$ & $0(0 \%)$ & $<0.01$ \\
AOS & $22(36.7 \%)$ & $5(12.2 \%)$ & \\
AOD & $0(0 \%)$ & $7(17.1 \%)$ & \\
with epidural anesthesia & $57(95 \%)$ & $26(63.4 \%)$ & $<0.01$ \\
with peripheral nerve block & $0(0 \%)$ & $4(9.8 \%)$ & 0.014 \\
with fentanyl administration & $14(23.3 \%)$ & $41(100 \%)$ & $<0.01$ \\
with remifentanil administration & $21(35 \%)$ & $36(87.8 \%)$ & $<0.01$ \\
\hline extubation at ICU (cases) & 17 & & \\
the very day of operation & 14 & & \\
after the very day of operation & 3 & & 0.684 \\
Re-intubation at ICU(cases) & 0 & 3 & \\
\hline
\end{tabular}

